\title{
Research on the Distribution System Simulation of Large Company's Logistics under Internet of Things Based on Traveling Salesman Problem Solution
}

\author{
Liu Hui, Chen Min
}

School of Computer Science and Technology, Hunan Institute of Technology, Hengyang, China Email:158138698@qq.com

Abstract: This paper is research on the distribution system's simulation of large company's logistics under Internet of Things (IoT) based on traveling salesman problem solution. The authors claim that the real-time traffic synergy is better than traditional distribution strategy in general, verifying and comparing the simulation results in different situations of goals and starting time distribution. The simulation method used in this paper makes the result more scientific and reliable. The simulation truly reflects the degree of influence of information synergy to improve the efficiency of logistics system. The information synergy had an obvious enhancing effect on system operation efficiency. Taking full advantage of the value of information and improving the information synergy to the best level have irreplaceable effect on system operation efficiency.

Keywords: Main simulation method, Distribution system, Large Company's Logistics, Internet of Things (IoT), Traveling salesman problem solution.

\section{Introduction}

With the rapid development of Internet of Things (IoT) technology, a huge business value network is formed. The results would encourage multiple service providers to develop services for IoT [1]. Multiple services providers will require the development of a suitable, scalable service delivery platform, which enables the fast and cost-effective creation of new IoT services. However, the network capacity of IoT has some characteristics of error-prone, unreliable, limited energy, and constrained resources, so how to deliver services over IoT becomes a big challenge in service computing. The research topic discussed in this paper is about IoT-based service delivery, which includes the service delivery framework model, IoT 
capabilities abstract framework and related algorithms, distributed service description, dynamic combination based on life cycle model and its algorithm, trustful service exposure, negotiation mechanism and its algorithm for multiple service providers. Based on TMF SDF, SensorLogic SDP, and L. Atzori IoT middleware, an IoT-based service delivery framework model is proposed for chaos of the IoT service environment, service development of "chimney", and lack of sustainability. The first, IoT-based service delivery environment is divided into service providers, service consumers, and IoT infrastructure providers by referring to TMF SDF.

Paper Du, Wang and Hong [2] prompts a model which realizes IoT resource share using dynamic coordination and abstraction technology. Lastly, complex service is quickly created by service dynamic composition. Compared with TMF SDF, SensorLogic SDP, and L. Atzori IoT middleware, the model effectively solves problem of IoT services stability, service dynamic composition, and trustful service exposure. Referred to EU FP7SENSEI, G. Fortina object abstraction and dynamic coordination, this paper proposes a novel IoT capabilities abstraction framework with dynamic collaboration, self-organization and fault tolerance, and its related algorithm. The framework of $\mathrm{Zhou}$ and $\mathrm{Ma}$ [3] firstly abstracts Smart Object in the Internet of things (CHN node) into PRA agent for the unstructured data real-time and complexity. This has intelligence formalization, selfmanagement ability, and autonomic computing ability for each PRA agent. Then, a RCT-based coordinate algorithm is proposed for each PRA limited computing power. Lastly, a TFA and Load-based self-evaluation algorithm is proposed for each PRA internal structure dynamic variable. Compared with EU FP7SENSEI, G. Fortina object abstraction and dynamic coordination, the model and some algorithm not only maintain IoT services stability and reliability, but also support the service dynamic composition. According to the E. G. da Silva dynamic service composition and S. C. Geyik robust dynamic sensor service composition, Y an g, P an g and $\mathrm{Zh}$ an $\mathrm{g}$ [4] propose a life cycle-based distributed service description and dynamic composition model and its algorithm for a large heterogeneity, unstructured datadriver service, and decentralization service composition of atomic sensor service. Each PRA is abstracted into semantic service by metadata in sensor service description, which enhances robustness of service. According to service life-cycle, the dynamic sensor service composition is divided into four phases: service planning, discovery, selection and execution. In the planning phase, it proposes generation algorithm of dynamic service composition for Planning Domain, User Requirement and Workflow. In the discovery phase, it proposes service discovery algorithm based on input/output semantic matching. In the selection phase, it has proposed service selection algorithm based on Fuzzy Logic and PSO. In the execution phase, dynamic service composition script is deployed on service broker, which service composition metadata is dynamically mapped on IoT network capabilities. Compared with E.G. da Silva dynamic service composition, and S. C. Geyik [5] robust dynamic sensor service composition, the model and some algorithm effectively solves problem of unstructured data-driver service dynamic composition. Referencing S. Alam service exposure of IoT services and T. Finin 
service exposure on SDP, in the paper of $\mathrm{Liu}$ and $\mathrm{Zhu}$ [6] is proposed the trustful service exposure and negotiation mechanism for multiple service providers. The mechanism firstly assigns virtual ID card to each service provider by trustbased access control. Then, the mutual trust is established by the direct or indirect trust reasoning, and the trustful service exposure mechanism is established by the trustful service level agreement negotiation. Finally through comprehensive SLA evaluator, service capabilities are assigned to service provider. Compared with S. Alam service exposure of IoT services and T. Finin service exposure on SDP, service delivery capabilities are exposed for multiple service providers by the trustful service exposure and negotiation mechanism.

\section{The framework of Traveling salesman problem}

The genetic algorithm and the ant colony optimization algorithm are studied seriously, then some new ideas and novel algorithms are proposed. These novel algorithms are successfully applied to the Traveling Salesman Problem (TSP) and distribution network reconfiguration. Based on the analysis of fitness landscape of TSP, a variable neighborhood search mutation operator, called GIIM, is designed by combining simple inversion mutation operator with insertion mutation operator. The GIIM can adaptively adjust the size of neighborhood search, and has very strong ability of local search. Based on GIIM, an efficient genetic algorithm for TSP, called EGA, is presented by combining Partially Matched crossover (PMX) and annealing selection with elitist strategy. The simulation for TSP shows that EGA has not only very strong global search ability but also very fast convergence speed, whose testing results are the same or even more superior ones in comparison with the optimal path in the newest literatures and TSPLIB. Based on the analyses of human evolution's properties, a novel Genetic Algorithm (GTGA) is proposed with analogies to the gene pool and two basis gene operation methods in gene therapy theory. The core of GTGA lies on construction of a gene pool and a therapy operator, which consists of insertion operation and removing operation. The methods of creation and updating of the gene pool and construction of the therapy operator are given and demonstrated by TSP. The theoretical analysis and simulation results of TSP show that GTGA can restrain the degeneration and premature convergence phenomenon effectively during the evolutionary process while greatly increasing the convergence speed [7].

TSP (Traveling Salesman Problem) is a combination optimization problem with simple definition but difficult to be solved, which attracts many researchers in various fields including mathematics, physics, biology and Artificial Intelligence (AI). It has become and will continue to be a standard problem to test new algorithms of combination optimization. Theoretically speaking, the enumeration not only can solve TSP, but also can get the best answer. But the best answer in such huge search space is hard to obtained by nowadays computers using common enumeration. Therefore, all kinds of algorithms to solve TSP emerged because of demand. Among of them, evolutionary algorithm is one of advanced technologies. Evolutionary Algorithm (EA) is an intelligent algorithm that learns from the 
evolutionary process in the nature. EA employs a coding technology and some genetic operations. Under the pressure of selection, which means "fits survive", the algorithm can produce an optimal solution. EA becomes a general solver of challenge problems, because it is simple and seldom needs any additional information about the problem. The new 2-adjacency representation of the Hybrid Evolutionary Algorithm (HEA) is presented in Jiang et al. [8]. The reason for this is that the traditional routing representations are not considerably appropriate for evolutionary processing. The new representation which is exclusive for each routing improves the heritability of evolutionary operators. The properties and operations of this new representation turn into the parameters and evolutionary operators of the HEA. The local optimizations are hybridized in, in order to speed up HEA. Local optimizations are the methods which are always used to solve the TSP. They are also parts of some other methods. They are very efficient. The performances of different local optimizations are analyzed in this paper. This paper presents a hybrid local optimization which is a part of the HEA. The experiments of TSP benchmarks indicate that the proposed scheme reaches the existing optimal solutions, even gets better solutions. Concerning the efficiency, hybrid local optimization has better efficiency, but the HEA is able to get better solutions with acceptable efficiency. A multi-threading HEA is implemented, in order to speed up the scheme. The results reveal the parallel essence of evolution algorithms [9].

The logistics in China has made a rapid evolution because of its connection to the world, and also because of the broken constraints of the external conditions. In $\mathrm{Han}$ et al. [10], some important problems of logistics are proposed and some of the solutions of these problems are offered and confirmed by simulations. The concepts of logistics are shown, the statistics of logistics in our country and abroad is analyzed. Then some of the important problems connected to design and implementations of logistics have been proposed. The choice of model for the problem to find the best route of a logistic problem is studied; a model based on M-TSP is chosen. The solution of an M-TSP is studied firstly, and then a typical logistic routine problem is transformed into an abstract and quantitative model based on M-TSP. An important combinatorial optimization problem, which is called TSP with NP-complete property, is solved by Ant Algorithm. In the latter part of this paper, simulations of some TSPLIB problems are shown to confirm the effectiveness of the algorithm. At last, in the paper $\mathrm{Han}$ et al. [10] are given some methods or advice on how to solve some of the other problems of logistics.

\section{Demand and information synergy type}

From the process of logistic, distribution has a strong demand of information synergy if compared to transportation or warehousing. In order information transmission, transportation monitoring, loading and unloading cargo information, sorting optimization attention is paid to the logistics information system of internal information synergy and reasonable operation. If the goods start distribution, client can track the goods from information system platform; logistics enterprise can control the goods distribution path real-time; transportation management 
department can monitor the vehicle movement real-time with a lot of internal and external information flow, real-time information processing and feedback. Therefore, we set the simulation model for distribution modules, where validation of real-time information has practical significance to enhance the efficiency of distribution.

An effective traveller information system has the potential to ease the impacts of incident conditions wide in the network. It is also important to note that the use of information may detriment some OD pairs while benefiting other OD pairs. The best fit distributions showed that a considerable number of drivers accepted smaller gaps during congested traffic conditions than in free flow conditions. A methodology is described that can be used to update the times in the bus timetables by using schedule adherence data. The goal of this methodology is to maximize the on-time density area.

In the city logistics distribution, right choose of route often determines total efficiency and cost of distribution process. With the development of city size, distribution of the distance between nodes is also widening; it is difficult to judge the optimal path in space intuitively. At the same time, with increasing city-auto possession, road resource is occupied and congestions change traffic conditions in peak time. These leads to difficulties in city logistics distribution to be judged the right times on the base of historical experience. Resent situation of city traffic is: expended road network, more complex road conditions, and more prediction errors of delivery time. Along with the further improvement of China's infrastructure and the progress of traffic data collection technology, the internal and external information synergy distribution modelling and simulation made a prominent contribution to improving distribution efficiency and reducing distribution cost.

Logistics distributions need to meet the following conditions: (1) logistics distribution operations standardization, (2) quick logistics distribution response, (3) more accurate data for the predicted market, (4) distribution process modernization. The demand of information synergy is more important than other logistic processes, because distribution uses a variety of information means and multiple ports keep real-time information collection and transmission.

The information synergy simulation model is as follows:

$$
\begin{aligned}
& \operatorname{TSP}(t)= \begin{cases}\mathrm{TSP}_{1}, & 0 \leq t \leq \Delta t, \\
\operatorname{TSP}_{2}, & \Delta t \leq t \leq 2 \Delta t, \\
\cdots & \\
\operatorname{TSP}_{n}, & (n-1) \Delta t \leq t \leq n \Delta t,\end{cases} \\
& \min d(T)=\sum_{k=1}^{n} \sum_{i=1}^{N} \sum_{j=1}^{N} c_{i, j}(k \bullet \Delta t), \\
& \text { s.t. } \Delta t=\frac{T}{n}, \frac{\Delta c_{i, j}}{\Delta t}=0 .
\end{aligned}
$$


Equation (1) shows that distribution problem based on information synergy was in the different sampling cycle of different TSP combinations. The problem turns into the traditional TSP when $\mathrm{TSP}_{i}$ are one and the same. This paper discusses cases with different $\mathrm{TSP}_{i}$. Equation (2) shows that the ultimate goal of distribution is the sum of all desired values of TSP during the $T$ time cycle. Its overall objective is optimization, not to pursue local optimum. Equation (3) is the definition of sampling cycle. It shows that in the sampling period of parameters remain unchanged.

We may get the calculating method for the main index in the following equations:

$$
\begin{gathered}
M_{i j}=\exp \left(-\frac{\left\|x_{i}-x_{j}\right\|^{2}}{\sigma^{2}}\right), \\
L=\left[\begin{array}{lll}
L_{1} & & \\
& \ddots & \\
& & L_{k}
\end{array}\right] .
\end{gathered}
$$

Their matching eigenvectors matrix is

$$
H=\left[h_{1}, h_{2}, \ldots, h_{k}\right]=A^{1 / 2} E .
$$

So, we can get:

$$
\begin{gathered}
U_{i j}=\frac{H_{i j}}{\sqrt{\sum_{t=1}^{k} H_{i t}^{2}}}, \quad i=1, \ldots, n, \quad j=1, \ldots, k, \\
P=I-A^{-1 / 2} M A^{-1 / 2} .
\end{gathered}
$$

According to the Equation (6), the calculating formula can be obtained in the next equations:

$$
\begin{gathered}
g(x, \omega)=\frac{1}{(2 \pi)^{3}} \int g(k, \omega) \exp (-i k \cdot x) d k, \\
g(k, \omega)=\left\|\begin{array}{ll}
G_{i k}(k, \omega) & \gamma_{i}(k, \omega) \\
\gamma_{k}^{\mathrm{T}}(k, \omega) & g(k, \omega)
\end{array}\right\|, \\
G_{i k}=\left(\Lambda_{i k}+\frac{1}{\lambda} h_{i} h_{k}^{\mathrm{T}}\right)^{-1}, g=-\left(\lambda+h_{i}^{\mathrm{T}} \Lambda_{i j}^{-1} h_{j}\right)^{-1}, \\
\gamma_{i}=\frac{1}{\lambda} h_{k}^{\mathrm{T}} G_{k i}, \\
\Lambda_{i k}(k, \omega)=k_{j} C_{i j k l}^{0} k_{k}-\rho_{0} \omega^{2} \delta_{i l}, \\
h_{i}(k)=e_{k i l}^{0} k_{k} k_{l}, h_{l}^{\mathrm{T}}=e_{i k l}^{0 \mathrm{~T}} k_{i} k_{k}, \\
\lambda(k)=\eta_{i k}^{0} k_{i} k_{k},
\end{gathered}
$$




$$
\frac{1}{2 \pi} \int_{-\infty}^{\infty} e^{-i k_{3} x_{3}^{\prime}} d x_{3}^{\prime}=\delta\left(k_{3}\right) .
$$

In traditional static simulation, we don't consider real-time traffic information synergy, and then weights of road depend on the length of road only. It means that the solution of the shortest path is got, and this result is not the real demand of distribution. Therefore, the distribution simulation system requires the road weights which considered real-time traffic condition information to ensure that the result of simulation has reality effect.

Congestion coefficient is the most important coefficient which reflects the real-time traffic condition information. The higher of congestion coefficient, the more road congestion, and the traveling time becomes longer. In order to reflect the change of congestion coefficient in different periods, we assume that one day has 2000 time units. According to morning and evening peaks, we divided the 2000 time units into 5 periods. During the simulation process, the start of distribution was marked with $t$. We calculate the average value of congestion coefficient during the $\mathrm{t}$ period. Calculation formula is listed below:

$$
v_{t}=(1-f) v_{0},
$$

where $v_{0}$ is vehicle frees speed (average speed of vehicle when there is no traffic congestion);

$v_{t}-$ vehicle speed, which is considered in the real-time traffic conditions;

$f$ - congestion coefficient.

When $f$ increases, vehicle speed decreases accordingly, the weight of the same road increases, and vice versa. The calculation formula of weight of road is

$$
\frac{\Delta c_{i, j}}{\Delta t}=0, \quad \Delta t=\frac{T}{n} .
$$

If the current time distribution task is not complete, we set the vehicle arriving last distribution goal as a starting point. According to the value $\mathrm{f}$ in the next period, weight of road is calculated from Equation (15), and the next road is chosen. When the deduction is repeated according to the above process, the final results of simulation will be achieved. Fig. 2 shows the structure of online logistics automatic monitoring system. Fig. 3 shows the network nodes for the logistics system.

As far as a person has history regularity of various traffic behaviours during the day, therefore, in different periods the city traffic is also different. The starting time of distribution also affects the overall effect of the distribution. We set the node number of cities as 10 , and initial velocity of distribution vehicle is 200 . In traditional simulation, because we do not consider real-time traffic information, we just think about the shortest route. So, the simulation result of whole time is longer than with considered information synergy. We can't find the best route because of that reason. 


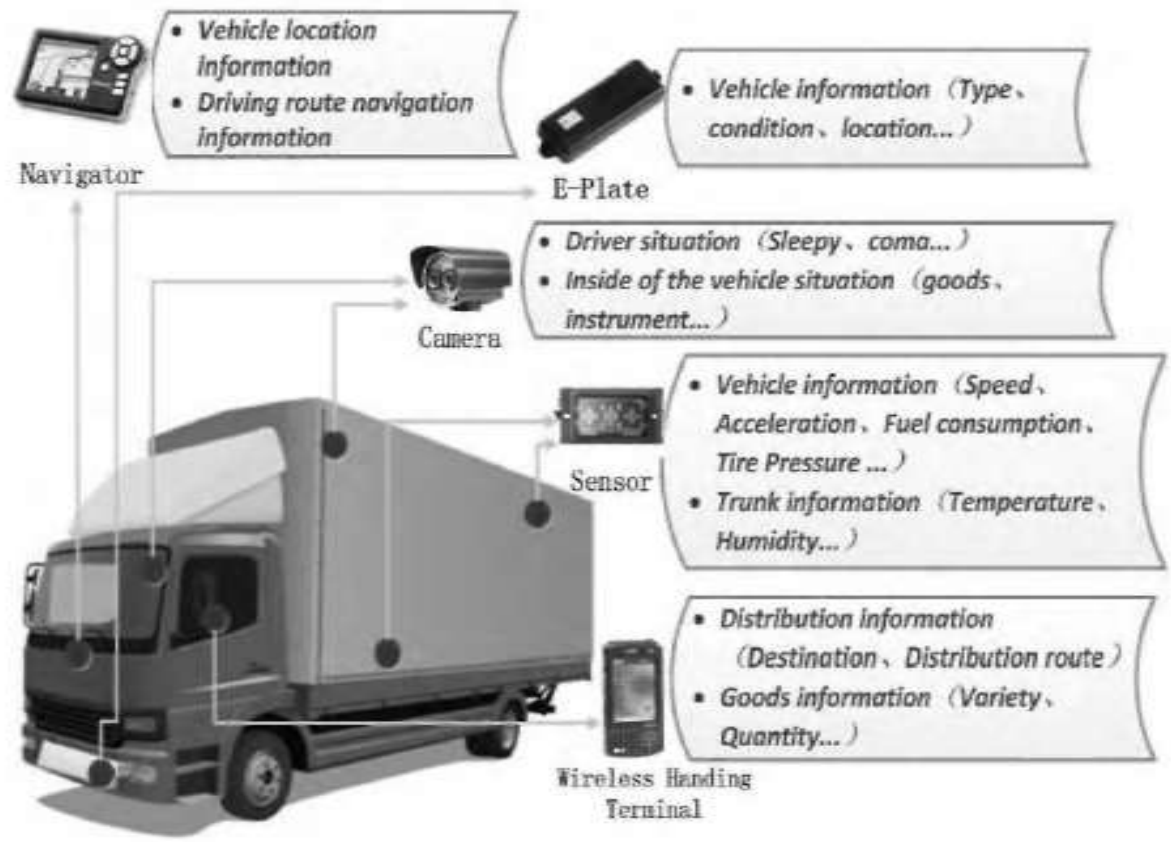

Fig. 1. The Information Synergy Type of the Truck includes Vehicle information, location information, Driver information, Truck information, tracking information and so on

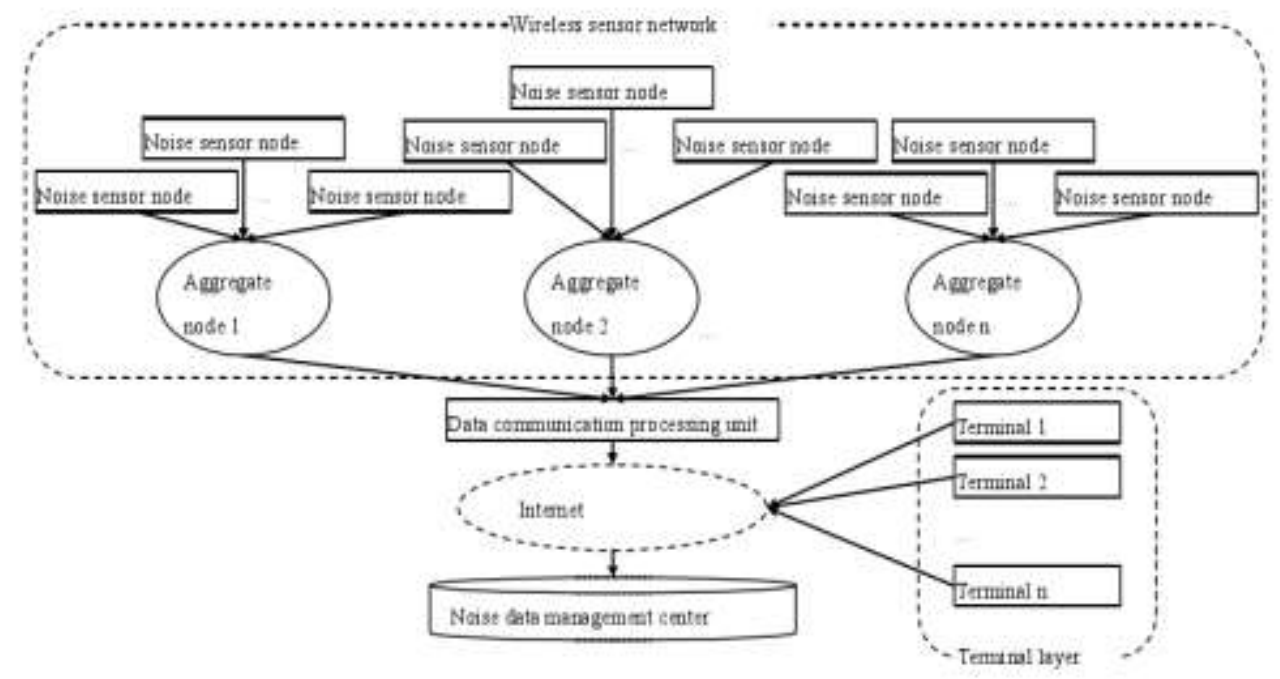

Fig. 2. The structure of online logistics automatic monitoring system 


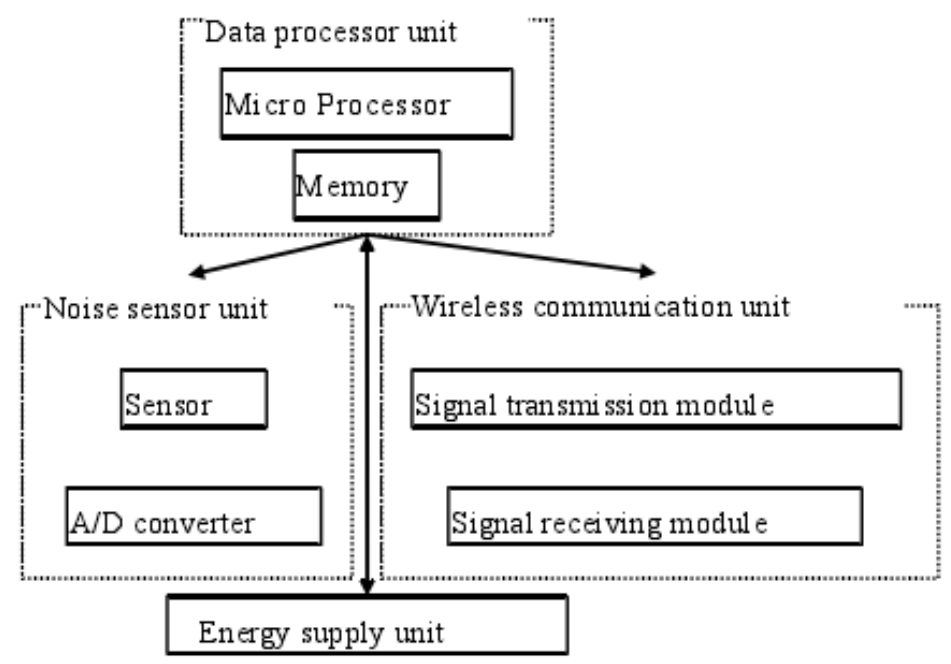

Fig. 3. The network nodes for the logistics system

However, the network capacity of IoT has some characteristics of error-prone, unreliable, limited energy, and constrained resources. So, how to deliver services over IoT becomes a big challenge in service computing. The research topic discussed in this paper is about IoT-based service delivery, which includes the service delivery framework model, IoT capabilities abstract framework and related algorithms, distributed service description and dynamic combination based on life cycle model and its algorithm, and trustful service exposure and negotiation mechanism and its algorithm for multiple service providers.

\section{Conclusion}

From the process of logistic, compare with order, transportation and warehousing, distribution has a strong demand of information synergy. Order information transmission, transportation monitoring, loading and unloading cargo information, sorting optimization attention is paid to the logistics information system of internal information synergy and reasonable operation. If the goods start distribution, client can track the goods from information system platform, logistics enterprise can control the goods distribution path real-time, transportation management department can monitor the vehicle movement real-time with a lot of internal and external information flow, real-time information processing and feedback. Therefore, we set the simulation model for distribution modules and validation of real-time information to enhance the efficiency of distribution to practical significance.

This paper demonstrated that information synergy can significantly improve the efficiency of distribution system. In this information synergy system, IOT technology has been applied. A simulation study has shown that with the synergy of real-time traffic information, customers can trace cargo information, logistics 
enterprise can real-time optimize distribution path, traffic management department can real-time monitor vehicle movements. The implications of the research are to enhance the modern logistics system efficiency and improve the level of distribution service. The IOT technology is the best method which enhanced the integration of city logistics and transportation information.

Acknowledgements: This work was financially supported by project of Hunan (No 2013SK3177) and project of Hengyang (No 2013KG68).

\section{References}

1. Al-Turjman, F. M., A. E. Al-Fagih, W. M. Als alih, H. S. Has s ane in. A DelayTolerant Framework for Integrated RSNs in IoT. - Computer Communications, 2013, pp. 369-373.

2. D u, K.-K., Z.-L. W a n g, M. H o n g. Human Machine Interactive System on Smart Home of IoT. - Journal of China Universities of Posts and Telecommunications, 2013, pp. 20-30.

3. Z h o u, M., Y. M a. QoS-Aware Computational Method for IoT Composite Service. - Journal of China Universities of Posts and Telecommunications, 2013, pp. 20-29.

4. Y a n g, J.-C., H. P a n g, X. Z h a n g. Enhanced Mutual Authentication Model of IoT. - Journal of China Universities of Posts and Telecommunications, 2013, pp. 205-221.

5. R o sári o, D, Z. Zh a o, A. S a n t os, T. B r a u n, E. Ce r que i r a. A Beaconless Opportunistic Routing Based on a Cross-Layer Approach for Efficient Video Dissemination in Mobile Multimedia IoT Applications. - Computer Communications, 2014, pp. 45-52.

6. L i u, S.-J., G.-Q. Z h u. The Application of GIS and IOT Technology on Building Fire Evacuation. - Procedia Engineering, 2014, pp. 71-80.

7. Ramakrishnan, A. K., D. Preuveneers, Y. B erbers. Enabling Self-Learning in Dynamic and Open IoT Environments. - Procedia Computer Science, 2014, pp. 32-40.

8. J i a n g, H., F. S h e n, S. C h e n, K.-C. Li, Y.-S. J e o n g. A Secure and Scalable Storage System for Aggregate Data in IoT. - Future Generation Computer Systems, 2014, pp. 122-129.

9. M a r t in h o, R., D. D o ming o s. Quality of Information and Access Cost of IoT Resources in BPMN Processes. - Procedia Technology, 2014, pp. 16-22.

10. H a n, K., S. L i u, D. Z h a n g, Y. H a n. Initially Researches for the Development of SSME under the Background of IOT. - Physics Procedia, 2012, pp. 24-30. 